Die Cholesterinbestimmung ist eine der häufigsten Untersuchungen der Laboratoriumsmedizin. Als klinisch bedeutsames Entscheidungskriterium werden nur die in der Literatur unterschiedlich angegebenen oberen Referenzwerte erachtet. Wenig Daten liegen über den unteren Referenzwert vor, noch weniger Kenntnisse über die klinische Bedeutung niedriger Cholesterinwerte. Deshalb wurde Herr Dr. Boskamp, der sich als Nichtmediziner seit Jahren mit dem Thema Hypocholesterinämie befaßt, gebeten, seine Vorstellungen darzulegen.

\title{
Die Cholesterin-Parabel und der Reigen der Sterine
}

\author{
E. K. M. Boskamp
}

Zusammenfassung:

Hypothese und Versuch eines Beweises für den kausalen Zusammenhang zwischen Fehlernährung und der Entstehung von Neoplasien, sklerotischen Vorgängen und Arteriosklerose. Die besondere Rolle der Sterine Cholesterin, Hormone, Gallensäuren - wird dargestellt. Diskussion der Hypocholesterinämie - im Gegensatz zur Hypercholesterinämie - als ursächlicher Indikator für Krebsentstehung. Vorschlag zur Verhinderung von Krebswachstum durch Ernährungsumstellung durch die beschriebenen Überlegungen.

Schlüsselwörter:

Hypocholesterinämie - Sterine - maligne Tumoren

\section{Summary:}

Hypothesis and attempt to prove the causal connection between malnutrition and genesis of neoplasms, sclerotic processes and arteriosclerosis. The special role of sterols - cholesterol, hormones, gallic acids, etc. - Discussion on hypocholesterolemia - in contrary to hypercholesterolemia - as a causal marker of cancer development. Proposal to stop cancer progression and initiate regression by changing dietic conditions.

Keywords:

hypocholesterolemia - sterols - neoplasm

\section{Einleitung}

Die Würdigung der Arbeiten von Michael S. Brown und Joseph L. Goldstein über den Cholesterinstoffwechsel durch Verleihung des Nobelpreises für Medizin 1985 wird die Diskussion um dieses Lipid erneut anfachen und dem Ruf nach Cholesterinspiegelerniedrigung neue publizistische Nahrung verleihen.

Während der Hypercholesterinämie in der medizinischen Literatur breitester Raum gewidmet wird, findet man den Begriff der Hypocholesterinämie ruur selten unter den Stichworten. Dabei ist ein menschliches Leben mit dem Cholesterinspiegel $0,0 \mathrm{mg} / \mathrm{dl}$ undenkbar, also muß es einen minimalen, das Leben noch ermöglichenden Wert geben, und in Annäherung an diesen Wert pathologische Zustände der Hypocholesterinämie.

In „Labor und Diagnose” (L. Thomas Medizinische Verlagsgesellschaft m.b.H., Marburg/Lahn 1978) heißt es lediglich im Hinblick auf die Arteriosklerose: "Nach Schettler und Nüssel Cholesterinwerte bis $220 \mathrm{mg} / \mathrm{dl}$ unauffällig, 220 bis $260 \mathrm{mg} / \mathrm{dl}$ verdächtig, ab $260 \mathrm{mg} / \mathrm{dl}$ erhöht." Auf eine Unterteilung in HDL und LDL soll hier nicht eingegangen werden, weil in dieser Veröffentlichung das Schwergewicht auf die niedrigen Gesamtplasmacholesterinwerte gelegt werden soll.

Es stellt sich die Frage: Welches sind die pathologischen Zustände der Hypocholesterinämie, und welches ist der optimale Wert für den Plasmacholesterinspiegel zwischen deń Extremen der zu hohen und zu niedrigen Werte? Der Verfasser ist der Auffassung, daß die derzeitigen Forderungen zur Cholesterinspiegelsenkung auf Werte unter $200 \mathrm{mg} / \mathrm{dl}$ bereits im pathologischen Bereich liegen, und daß der optimale Wert zwischen 260 und $280 \mathrm{mg} / \mathrm{dl}$ entsprechend 6,734-7,252 mmol/l liegen.

Es wird die Hypothese aufgestellt:

Hypocholesterinämie - Hypotonie - Krebsgeschehen Hypercholesterinämie - Hypertonie - Arteriosklerose

Die in Abb. 1 dargestellte Cholesterin-Parabel zeigt die Krankheitsprogression mit fallendem und mit steigendem Plasmacholesteringehalt. Hiernach sollten vom Optimum bei 260 bis $280 \mathrm{mg} / \mathrm{dl}$ ausgehende fallende Cholesterinwerte prognoserelevante Indikatoren für die Progression des Krebsgeschehens sein, während umgekehrt, stei- 
gende Werte vom Optimum ausgehend zunehmende arteriosklerotische Veränderungen indizieren würden.

Diese Veröffentlichung soll dazu auffordern, die aufgestellte Hypothese zu überprüfen. Sie soll weiter ermutigen, zu versuchen, mit diätetischen Maßnahmen über eine Cholesterinspiegelerhöhung bis zum prognostizierten Optimun das Tumorgeschehen in Richtung Regression zu beeinflussen. Liegt in der Änderung von Ernährungsgewolınheiten möglicherweise die Erklärung für die in der Literatur beschriebenen spontanen Remissionen (1)?

In den Veröffentlichungen „Der Reigen der Sterine” (2) und ,Hypothese über den kausalen Zusammenhang zwischen menschlicher Ernährung und neoplastischen und sklerotischen Vorgängen" (3) sind die Beobachtungen und Literaturangaben zusammengefaßt, auf denen die vorgestellte Hypothese basiert. Hier sei das Wichtigste wiederholt und einige Tatsachen sind ergänzt.

\section{Tierexperimentelle Versuche zum Beweis des Zusammenhanges zwischen Mangelernährung und der Entstehung von Neoplasien}

Schon 1926 fand Fujimaki (4), daß bei Ratten Magenkarzinome durch eine A-Vitamin-arme Nahrung erzeugt werden können. In den Jahren 1922-1928 erschienen mehrere Arbeiten über die Zusammenhänge von A-Vitaminmangel und metaplastischen Veränderungen an den Epithelien des Respirations-, Gastrointestinal- und Urogenitaltraktes $(5-7)$. Mehrere Arbeiten zeigten, daß bei den Tieren durch Vitamin A die Entstehung sowohl präcanceröser Veränderungen wie auch von Papillomen und Plattenepithelkarzinomen verzögert oder verhindert werden können (8).

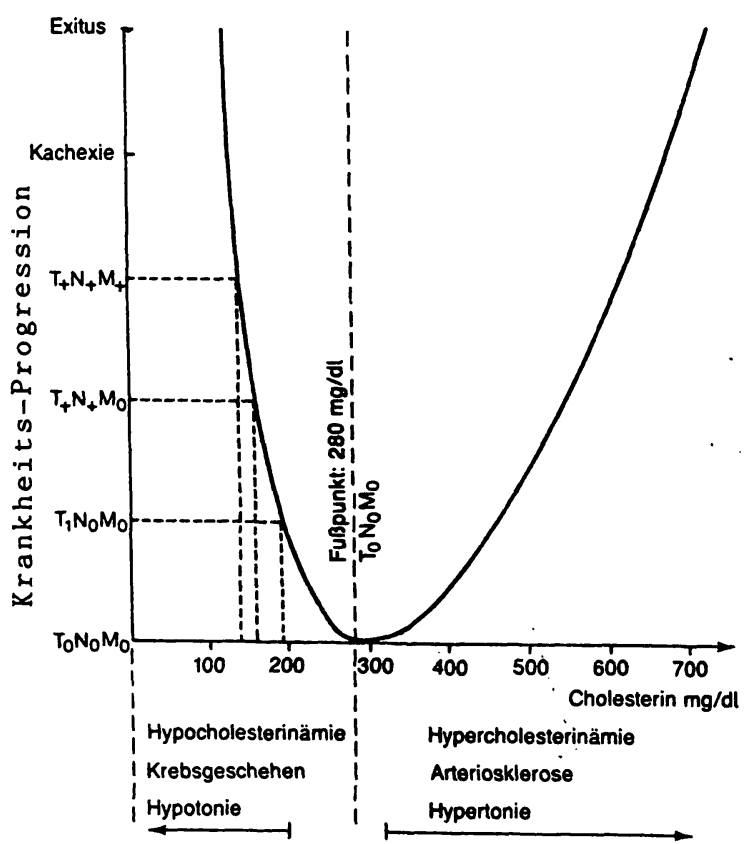

Abb. 1: Cholesterin-Parabel
1931 veröffentlichte Abderhalden „Studien über den Einfluß bestimmter Nahrungsarten auf den Gesamtzustand des tierischen Organismus" (9). Auf Seite 160 berichtet er über Langzeiternährung von Ratten mit Milchreis. Den Endzustand der Tiere beschrieb er folgendermaßen: „Bemerkenswert ist, daß ein auffallend hoher Prozentsatz der mit Milchreis ernährten Tiere Tumore aufwiesen. Es entwickelten sich zum Teil, ohne daß an den Tieren besondere Erscheinungen bemerkbar waren, große Geschwülste, vor allen Dingen in der Lunge. In anderen Fällen ging der Tumor vom Mesenterium aus und in wieder anderen war die Harnblase oder die Niere Ausgangspunkt für die Geschwulst. Von 86 Ratten, die $1 \frac{1}{2}$ bis $2 \frac{1}{2}$ Jahre im Versuch waren, zeigten 17 Geschwulstbildungen. Bei weiteren 8 war die Diagnose unsicher, d.h. es war ein kleiner Tumor zumeist in der Leber vorhanden."

In diesen Versuchen wurden als Nebenerscheinungen beobachtet: Blasensteine, Hautveränderungen, Veränderungen an den Augen, Verhornungen der Harnwege und der Trachea, Schwellungen der Hüft- und Kniegelenke, Lähmung der hinteren Extremitäten, anämische Zustände und Verhaltensveränderungen. Weiter wurden Versuche mit Milch und Weißbrot durchgeführt (S.165): „Von 12 Ratten, die nur Milch und Weißbrot erhielten, zeigten 3 kleine Tumore, und zwar handelte es sich um Spindelzellsarkome. Ausgangspunkt war zweimal das Mesenterium. In einem Fall saß der Tumor in der Leber. In einem weiteren Fall war eine in ihrer Art nicht vollkommen geklärte Wucherung vorhanden."

Dabei wurden Geschwulste nur dann erkannt, wenn sie ohne weiteres makroskopisch festgestellt wurden. Einige Tumore wurden histologisch untersucht: z. B. „Spindelzelliges Mesenterialsarkom auf die Leber übergreifend. Die Obduktion ergab weiter: Milz vergrößert, Nieren pseudomelanotisch, Knochen außerordentlich brüchig, Nebennieren vollkommen fettfrei."

Als Nebenerscheinungen wurden beobachtet: Blasensteine, Geschwüre im Darmkanal und Blutungen, es wird auch auf eine Spasmophilie hingewiesen.

Bemerkenswert ist es, daß in den letzten Versuchen bei Zufütterung von Lebertran und Trockenhefe keine Tumorbildung beobachtet wurde. Auch bei Verfütterung von Weizenschwarzbrot traten Störungen in vermindertem Umfang auf.

Zusammenstellung der Versuchsergebnisse

\begin{tabular}{lllll}
\hline $\begin{array}{l}\text { Art der } \\
\text { Ernährung }\end{array}$ & $\begin{array}{l}\text { Ratten } \\
\text { im Ver- } \\
\text { such }\end{array}$ & $\begin{array}{l}\text { Tumore } \\
\text { ein- } \\
\text { deutig }\end{array}$ & $\begin{array}{l}\text { un- } \\
\text { sicher }\end{array}$ & $\begin{array}{l}\text { Tumor- } \\
\text { häufig- } \\
\text { keit (\%) }\end{array}$ \\
\hline Milchreis & 86 & 17 & 8 & $19-29$ \\
Milchbrot & 12 & 3 & 1 & $25-33$ \\
\hline
\end{tabular}

Demgegenüber wird die Häufigkeit von Spontantumoren bei Ratten mit 3,4\% angegeben. (Nach Arbeiten von Guerin, 1954, in K. H. Bauer "Das Krebsproblem”, 1963. S.89.)

Aus'diesen Versuchen ergibt sich, daß mit bestimmten Mangelernährungen ohne Anwendungen irgendwelcher Carcinogene bei Versuchstieren mit hoher Häufigkeit Tumore in unterschiedlichen Organen entstehen, und zwar nicht nur bei unspezifischen Mangeldiäten, sondern insbesondere bei Versuchen zur Erzeugung von A-Avitaminosen. 
200. UND PHYTOSTEAINE
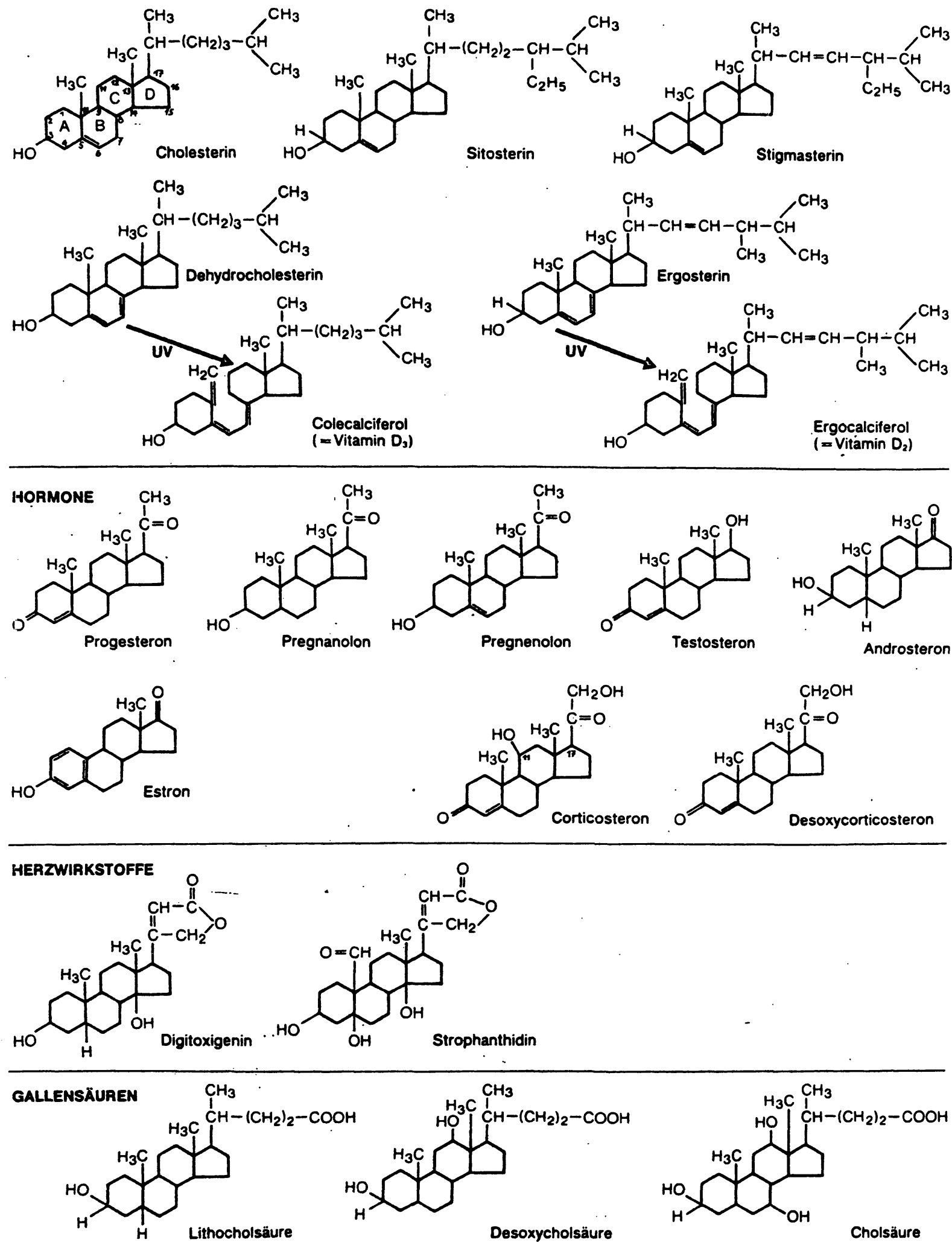
Die Vitamine $A$ und $D$ und die Bedeutung der Sterine

Vitamin A ist ein fettlösliches Vitamin und kommt speziell in den unverseifbaren Anteilen der Fette vor. Den Ausschluß des Vitamin $A$ aus einer Ernährung erreicht man im Versuch durch Verwendung von Fetten mit minimalen Anteilen an Unverseifbarem oder nach Abtrennung der unverseifbaren Anteile aus den Fetten. Mit dieser Abtrennung wird aber nicht nur das Vitamin A abgetrennt, sondern sämtliche fettlösliche Lipide, insbesondere auch die Sterine (Abb. 2).

Vitamin D ist heute der einzige Stoff aus der Sterinklasse, der in handelsüblichen, synthetischen Vitaminpräparaten Verwendung findet, der zusätzlich zur Vitaminierung vieler Nahrungsmittel gebraucht wird. Das antirachitisch wirksame Vitamin $D$ entsteht in vivo durch Sonneneinstrahlung aus 7-Dehydrocholesterin in der Haut und in vitro aus Ergosterin unter UV-Einstrahlung. Bei diesen Reaktionen wird der Sterinskelett aufgespalten, und es entsteht ein chemisch höchst aktives Trien, das mit den Sterinen nur nach der Herkunft verbunden ist. Nach seiner Entstehung in der Haut muß dieses Vitamin eigentlich als Hormon bezeichnet werden. Die Sterine sind eine chemische Stoffklasse, dessen Grundgerüst aus drei kondensierten C6 Ringen und einem C5 Ring aufgebaut sind. Die in der Natur vorkommenden Sterine unterscheiden sich durch Anzahl der Doppelbindungen im Molekül und unterschiedliche Substituenten an den einzelnen C-Atomen. Nach ihrem Vorkommen im Pflanzen- und Tierreich unterscheidet man Phytosterine und Zoosterine. Die ganze Körperklasse gehört zu den Lipiden, das sind Begleitstoffe der pflanzlichen und tierischen Fette, die unverseifbar sind und sich bei der Verseifung von Fetten im unlöslichen anreichern - wie auch das Vitamin A.

Abb. 2 zeigt einen unvollständigen Überblick wichtiger Sterine, die Phytosterine - Sitosterin - Stigmasterin Ergosterin, das wichtigste Zoosterin - das Cholesterin und die Sexualhormone, die Nebennierenrindenhormone (von letzteren sind allein über 20 verschiedene chemisch aufgeklärt und synthetisiert), therapeutisch genutzte Pflanzenwirkstoffe - Digitoxingenin - Strophantidin und die verschiedenen Gallensäuren.

Die Gallensäuren leiten mit ihren physikalischen und chemischen Eigenschaften die Fettverdauung im Organismus ein. Sie sind Endprodukte des Sterinstoffwechsels.

Letzteren kann man sich im Prinzip folgendermaßen vorstellen: Phyto- und Zoosterine werden mit der Nahrung aufgenommen. Das Cholesterin dient als wichtiger Baustein in jeder Zelle. In entsprechend spezialisierten Zellbereichen werden bei genügendem Angebot Sterine umgebaut zu den vielen bekannten Wirkstoffen, deren Gesamtzahl und deren Genese in Einzelheiten weitgehend unbekannt sind. Die Endprodukte des Sterinstoffwechsels, die Gallensäuren, werden mit anderen Sterinen aus der Galle in das Duodenum ausgeschieden und sind Voraussetzung für die Resorption mit der Nahrung aufgenommener Fette. Ein Teil der Gallensäuren wird aus dem Verdauungsbrei rückresorbiert und der Leber im enterohepatischen Kreislauf wieder zugeführt.

Dieses vielgestaltige, in allen Einzelheiten unfaßbare und nur in Andeutung erkennbare lebensregulierende $\mathrm{Ge}$ schehen habe ich als den "Reigen der Sterine" beschrieben, um seine besondere Bedeutung zu betonen.
Rückresorption und Entstehung von Neoplasien

Die Rückresorption im enterohepatischen Kreislauf wird sich bei einer Verarmung des Organismus an Cholesterin, also bei fallenden Plasma-Cholesterin-Werten, zum Ausgleich der hierdurch hervorgerufenen Mangelsituation verstärken. Gleichzeitig wird durch die erhöhte Rückresorption auch ein Anstieg der Gallensäurenkonzentrationen und des Bilirubins im Blutplasma zu beobachten sein.

Die Rückresorption wird effektiver durch längere Verweildauer des Verdauungsbreis im Darm (Obstipation) und mit Vergrößerung der Austauschflächen. So stellt sich die Frage, ob Polypen und Divertikel im Verdauungstrakt ihre Entstehung der Aufgabe verdanken, durch Vergrößerung der Oberflächen die Rückresorption zu aktivieren.

Dient möglicherweise auch das Blasenpapillom als Kunstorgan zur Rückresorption von Flüssigkeit und wasserlöslichen Komponenten aus dem Urin und ist damit ein Symptom für eine Mangelsituation?

\section{Hypocholesterinämie und Krebsgeschehen}

Die älteste, mir bekannte Arbeit zu diesem Thema „Beitrag zur Frage des Fettstoffwechsels bei Krebskranken" von Klaus Karel aus der I. Frauenklinik und dem Institut für Medizinische Chemie der Karl-Universität in Prag wurde im Jahre 1928 veröffentlicht (10).

Hieraus zitiere ich auszugsweise wörtlich:

„Diese Arbeit befaßt sich mit der Frage des Cholesterins und seiner Wechselbeziehung mit Fettsäuren im Blutserum von Frauen, welche am Krebs des Genitalapparates leiden ... Eine auffallende Tatsache ... ist eine deutliche Verminderung des gesamten Cholesterins im Serum der Krebskranken ... Aber auch im Anfangsstadium ... tritt eine deutliche Cholesterinverminderung zutage... Unsere Beobachtungen bestätigen analoge Befunde anderer Autoren, welche auch bei Carcinomen anderer Organe eine Hypocholesterinämie festgestellt haben ... Noch interessanter, aber schwer zu erklären ist eine hohe Cholesterinminderung bei fortgeschrittenen Carcinomen. In diesen Fällen, in welchen sicher große Massen von Krebszellen dem Zerfall anheimfallen, wäre es logisch, eher an eine Cholesterinanhäufung im Blute zu denken. Und gerade in diesen Fällen begegnen wir einer ausgesprochenen Hypocholesterinämie. Wie diese Hypocholesterinämie, welche sich von der durch Röntgenstrahlen hervorgerufenen Hypercholesterinämie so markant unterscheidet, zu deuten ist, läßt sich vorläufig nicht entscheiden ... Bei den ausgesprochen bösartig verlaufenden Prozessen begegnen wir einer starken Abnahme des Gesamtcholesterins, einer deutlichen Vermehrung von Fettsäuren und einem auffallend niedrigen lipämischen Koeffizienten ..." ?

Eine kürzlich im DKFZ in Heidelberg veranlaßte Computerrecherche Nr.282210 mit dem Titel „Cholesterinspiegel und Krebsgeschehen" bestärkte die Vermutung des Zusammenhanges - Hypocholesterinämie = Krebsgeschehen -, z. B mit der Arbeit „Serum-Cholesterol and 
Human Colon Cancer" Seth R. Miller et al. (11). Ich zitiere in Englisch: "Abstract-Comparison of serum cholesterol levels in a matched case-control study indicates that patients with colon cancer have serum cholesterol levels lower than those of controls. In 133 pairs matched by age and sex, serum cholesterol levels were $188 \pm 42 \mathrm{mg} / \mathrm{dl}$ for cases and $213 \pm 42 \mathrm{mg} / \mathrm{dl}$ for controls ... Matching of 130 early tumors to advanced tumors showed that women, but not men, had a significantly lower serum cholesterol level with advancing disease."

In den Mitteilungen einer internationalen Studiengruppe "Cholesterin und Krebstod" (12) wird im internationalen Bereich von niedrigeren Cholesterinwerten in Krebsfällen als bei Vergleichsfällen berichtet.

In der Arbeit "Review of the Epidemiological Evidence for a possible Relationship between Hypercholesterolemia and Cancer" von Manning Feinleib (13) heißt es:

"In this brief review I have attempted to document that the evidence relating hypercholesterolemia to an increased risk of cancer is controversal ..."

Die in der Literatur häufig zu findende Feststellung der hohen Krebshäufigkeit in Populationen mit hohem Fettverzehr und damit durchschnittlich hohen Cholesterinwerten, entstammen epidemiologisch-statistischen Vergleichsstudien, und werden widerlegt durch die Beobachtung der bei Krebskranken mit der Progression von Beginn bis zur Kachexie und zum Tod fallenden Cholesterinwerte. Es muß bedacht werden, daß die Ursachen für Krebs kein statistisch faßbares Bevölkerungsproblem sind, sondern Einzelschicksale der betroffenen Individuen, und somit statistische Erhebungen zu Fehlschlüssen führen können.

\section{Statistischer Überblick}

Trotz der generellen Skepsis in der Beurteilung statistischer Überlegungen sollen hier zwei statistische Tatsachen angeführt werden.

Tab. 1 zeigt - WHO "World Health Statistique Annual 1981" - die Krebshäufigkeit als Todesursache für 42 verschiedene Länder der Welt. Obwohl die WHO-Statistik auch eine Aufgliederung in die verschiedenen Carcinomarten enthält, bringt diese Übersicht die Daten der $\mathrm{Zu}$ sammenfassung .Position 08 - 14 malignant neoplasms, male and female all ages“. Diese Betrachtungsweise erscheint entkomplizierend und sachlich berechtigt, solange man weder für die einzelnè Art noch für die Gesamtheit der Krebsarten die Ursachen kennt.

Die Todesursache „Krebs” steigt demnach an von Nicaragua 14,7, Ägypten 19,9. Thailand 21,0 bis auf (BRD 252,8), England 260,7, Dänemark 266,1, UK-Schottland 269,2, jeweils Sterbefälle pro 100000 Einwohner.

Tab. 2 nach E. Gersfeld (14) zeigt einen ähnlichen Anstieg der Todesursache "Krebs" für Deutschland über die Jahre 1890 bis heute. Eine ähnliche Entwicklung ist auch für Amerika bekannt. Die Ursache für diese parallel verlaufenden Anstiege über die Zeit und in der ersten Tabelle über verschiedene Länder, dürfte weniger in der Gesamtindustrialisierung (z. B. Japan liegt verhältnismäßig günstig), als in der industriellen Bearbeitung von wichtigen Grundnahrungsmitteln zu suchen sein.

\section{Hypocholesterinämie - Ursache oder Wirkung?}

Diese Frage stellte bereits Karel (10) und sie ist bis heute nicht entschieden, bedauerlicherweise noch nicht einmal systematisch bearbeitet. Die eingangs zitierten Tierversuche (4-9) mit der Beobachtung der gehäuften Entstehung von Tumoren in Mangelsituationen ohne jegliche Carcinogene spricht für einen ursächlichen Zusammenhang. Die Entscheidung dieser Frage kann endgültig nur durch Therapieversuche erfolgen.

Tab. 1: Todesursache Krebs nach „World Health Statistique Annual 1981"

Deaths according to cause, death rates per 100000 population for 1979 if not special indicated.

Position 08 - 14 malignant neoplasms, male and female all ages

\begin{tabular}{|c|c|c|c|c|}
\hline & & & $\begin{array}{l}\text { Average } \\
\text { age }\end{array}$ & $\begin{array}{l}\text { Death } \\
\text { all ages }\end{array}$ \\
\hline $\begin{array}{l}1 . \\
2 . \\
3 . \\
4 . \\
5 . \\
6 . \\
7 . \\
8 . \\
9 . \\
10 . \\
11 . \\
12 . \\
13 . \\
14 . \\
15 . \\
16 . \\
17 . \\
18 . \\
19 . \\
20 . \\
21 . \\
22 . \\
23 . \\
24 . \\
25 . \\
26 . \\
27 . \\
28 . \\
29 . \\
30 . \\
31 . \\
32 . \\
33 . \\
34 . \\
35 . \\
36 . \\
37 . \\
38 . \\
39 . \\
40 . \\
41 . \\
42 .\end{array}$ & $\begin{array}{l}1976 \\
1977 \\
1978 \\
1978\end{array}$ & $\begin{array}{l}\text { UK + Schottland } \\
\text { Dänemark } \\
\text { England + Wales } \\
\text { Österreich } \\
\text { Ungarn } \\
\text { Bundesrepublik } \\
\text { Schweden } \\
\text { Luxemburg } \\
\text { Frankreich } \\
\text { Schweiz } \\
\text { Norwegen } \\
\text { Niederlande } \\
\text { Uruguay } \\
\text { Italien } \\
\text { Nordirland } \\
\text { Irland } \\
\text { USA } \\
\text { Finniand } \\
\text { Neu-Seeland } \\
\text { Argentinien } \\
\text { Australien } \\
\text { Bulgarien } \\
\text { Spanien } \\
\text { Island } \\
\text { Japan } \\
\text { Hongkong } \\
\text { Singapur } \\
\text { Cuba } \\
\text { Chile } \\
\text { Costa Rica } \\
\text { Paraguay } \\
\text { Venezuela } \\
\text { Suriname } \\
\text { Mauritius } \\
\text { Mexiko } \\
\text { Equador } \\
\text { Fidji } \\
\text { Guatemala } \\
\text { Domin. Republ. } \\
\text { Thailand } \\
\text { Ägypten } \\
\text { Nicaragua } \\
\text {. }\end{array}$ & $\begin{array}{l}71 \\
74 \\
73 \\
72 \\
70 \\
73 \\
75 \\
72 \\
74 \\
71 \\
75 \\
75 \\
70 \\
73 \\
71 \\
72 \\
73 \\
73 \\
72 \\
74 \\
71 \\
74 \\
76 \\
74 \\
74 \\
71 \\
69 \\
74\end{array}$ & $\begin{array}{l}269,2 \\
266,1 \\
260,7 \\
256,1 \\
251,6 \\
(252,8) \\
246,8 \\
239,9 \\
232,9 \\
222,2 \\
218,5 \\
215,2 \\
212,8 \\
201,9 \\
196,6 \\
190,0 \\
184,2 \\
183,1 \\
168,8 \\
154,4 \\
153,5 \\
151,7 \\
150,1 \\
140,9 \\
135,7 \\
126,6 \\
105,3 \\
101,2 \\
100,2 \\
69,5 \\
57,9 \\
55,1 \\
53,9 \\
46,8 \\
38,6 \\
37,9 \\
32,8 \\
28,7 \\
23,8 \\
21,0 \\
19,9 \\
14,7\end{array}$ \\
\hline
\end{tabular}

Tab.2: Anstieg der Todesursache Krebs nach E. Gersfeld (1950) (14)

1890 Krebs bei jedem 38 . Sterbefall die Todesursache 1900 Krebs bei jedem 30 . Sterbefall die Todesursache 1910 Krebs bei jedem 18. Sterbefall die Todesursache 1920 Krebs bei jedem 15. Sterbefall die Todesursache 1930 Krebs bei jedem 8 . Sterbefall die Todesursache 1950 Krebs bei jedem 6 . Sterbefall die Todesursache 
Für die Entstehung von Tumoren werden einige menschliche Ernährungsgewohnheiten und die industrielle Bearbeitung wichtiger Grundnahrungsmittel verantwortlich gemacht, die u. a. einen systematischen Ausschluß wichtiger Lipide bewirken.

1. Nach der modernen Vermahlung des Korns (eingeführt seit etwa 1900) enthalten nur noch $3 \%$ der Endprodukte für die menschliche Ernährung den Keim. Der Keim ist ein Hauptvorkommen der Sterine, wichtiger Lipide, z.B. Vitamin E und Proteine.

2. Bei der industriellen Fettherstellung zu Speiseölen und Margarine werden im Verlauf der Raffination Sterine und andere wichtige Lipide zum größten Teil entfernt.

3. Maßnahmen, die zur Erniedrigung der Plasma-Cholesterin-Werte empfohlen werden, insbesondere das Vermeiden tierischer Fette und tierischen Eiweißes (Innereien etc.), die besonders reich an Sterinen sind.
Schriftum:

1. THEISS. E.: Z. Alig. Med. 58, 1218 (1982)

2. BOSKAMP, E. K. M.: schwerpunkt med. 5. 5.69 (1982).

3. BOSKAMP, E. K. M.: schwerpunkt med. 6. 7.58 (1984)

4. FUJIMAKI, Y. P.: Cancer Res. 10, 469-477 (1926).

5. MORI, S.: Bull John Hopkins Hosp. 33. 357-359 (1922).

6. WOLBACH, S. B., HOWE, P. R.: J. Exp. Med. 42 (1925).

7. WOLBACH, S. B., HOWE, P. R.: Arch. Pathol. 5, 239-253 (1928).

8. WEBER, HARTMANN, ORBRECHT: Münchn. Med. Wschr. 124, 29 (1982).

9. ABDERHALDEN, E.: Biochem. Zeitschrift 234, 142-169 (1931).

10. KAREL, K.: Biochem. Zeitschrift Bd. 201, 286-291 (1928).

11. SETH R. MILLER: INCJ Vol.67, No.2, 297-300 (1981).

12. Cholesterin und Krebstod, JAMA D 2 (17), 821-828 (1983)

13. MANNING FEINLEIB: Cancerresearch (SUppl.) 43. 25033 25079 (May, 1983)

14. BAUER, K. H.: Das Krebsproblem, Springer-Verlag, S.52 (1963).

Anschrift des Verfassers:

Ernst Kurt Maria Boskamp

Dipl.-Chem. Dr. rer. nat.

Mary's Haus

2270 Oldsum 72 